\title{
Synthesis of natural products from the Indian neem tree Azadirachta indica
}

\section{Gemma E. Veitch, Andrea Pinto, Alistair Boyer, Edith Beckmann, James C. Anderson, Steven V. Ley*}

${ }^{1}$ H NMR Spectra were recorded on a Bruker DPX-400 (400 MHz), Avance 500 Cryo $(500 \mathrm{MHz})$ or a Bruker DRX-600 $(600 \mathrm{MHz})$ spectrometer using the deuterated solvent as internal deuterium lock. Chemical shift data are given in units $\delta$ relative to residual protic solvent where $\delta$ (chloroform $)=7.26 \mathrm{ppm}$. The multiplicity of a signal is indicated as: br - broad, $\mathrm{s}$ - singlet, $\mathrm{d}$ - doublet, $\mathrm{t}$ - triplet, $\mathrm{q}$ - quartet, $\mathrm{m}$ - multiplet, $\mathrm{dd}$ - doublet of doublets, $\mathrm{dt}$ - doublet of triplets, etc. Coupling constants $(J)$ are quoted in $\mathrm{Hz}$ and recorded to the nearest $0.1 \mathrm{~Hz} .{ }^{13} \mathrm{C}$ NMR Spectra were recorded on a Bruker DPX-400 (100 MHz), Avance 500 Cryo (125 MHz) or a Bruker DRX-600 $(150 \mathrm{MHz})$ spectrometer using the deuterated solvent as internal deuterium lock with broadband proton decoupling. Chemical shift data are given in units $\delta$ relative to residual protic solvent where $\delta$ (chloroform $)=77.0 \mathrm{ppm}$, t. NMR Spectra were assigned using information ascertained from DEPT, COSY, HMBC, HMQC and NOE experiments. IR spectra were recorded on a Perkin-Elmer Spectrum 1 FTIR spectrometer. The samples were prepared as thin films. Only selected absorbances $\left(v_{\max }\right)$ are reported. Optical Rotation was measured using a Perkin-Elmer Polarimeter 343 , the sample temperature was maintained at $25^{\circ} \mathrm{C}$ and $[\alpha]_{D}$ values are quoted in units of $10^{-1} \mathrm{~cm}^{2} \mathrm{~g}^{-1}$. High resolution mass spectra (HRMS) were recorded on a Waters Micromass LCT Premier spectrometer. Flash column chromatography was carried out using silica gel [Merck 9385 Kieselgel 60 (230-400 ASTM)]. Analytical TLC was performed on $0.25 \mathrm{~mm}$ thickness plates pre-coated with Merck Kieselgel 60 $\mathrm{F}_{254}$ silica gel. Where appropriate, reactions were performed using oven-dried glassware under an argon atmosphere. Reaction mixture composition was monitored by analytical TLC. Dry tetrahydrofuran (THF) was distilled from sodium in a recycling still using benzophenone ketyl as indicator; and toluene was freshly distilled from calcium hydride before use. Other solvents, $\mathrm{N}$-bromosuccinimide, silver oxide were purified using standard techniques. Other reagents were used as received. Ether refers to diethyl ether, hexane refers to $n$-hexane and petrol refers to petroleum distillate collected between $40{ }^{\circ} \mathrm{C}$ and $60{ }^{\circ} \mathrm{C}$. 
Common Intermediate 1

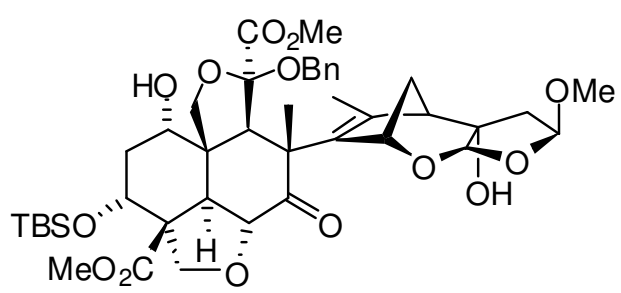

1 common intermediate

$[\alpha]_{\mathrm{D}}=+8.2\left(c=0.145, \mathrm{CHCl}_{3}\right) ; v_{\max } / \mathrm{cm}^{-1} 2950,1725,1453,1092,841 ; \delta_{\mathrm{H}}(600 \mathrm{MHz}$, $\left.\mathrm{CDCl}_{3}\right)$ 7.36-7.20 (10H, Ph), 5.30 (1H, dd $J$ 6.2, 4.2, H-23), 5.10 (1H, br.s, H-21), 4.98 (1H, d J 11.6, CHHPh), 4.80 (1H, br s, H-15), 4.50 (1H, d J 11.6, CHHPh), 4.44 (1H, d $J$ 11.6, CHHPh), 4.43 (1H, br s, H-1), 4.38 (1H, d J 11.6, CHHPh), $4.22(1 \mathrm{H}, \mathrm{d}$ $J$ 14.3, H-6), $3.84(1 \mathrm{H}, \mathrm{d} J$ 4.6, H-28), 3.82-3.77 (2H, H-3 + H-28), $3.80(3 \mathrm{H}, \mathrm{s}$, $\mathrm{CO}_{2} \mathrm{Me}$ ), 3.55 (1H, d J 9.3, H-19), 3.52 (1H, s, H-9), 3.48 (1H, d J 9.3, H-19), 3.42 $\left(3 \mathrm{H}, \mathrm{s}, \mathrm{CO}_{2} \mathrm{Me}\right), 3.41(3 \mathrm{H}, \mathrm{s}, \mathrm{OMe}), 3.10(1 \mathrm{H}, \mathrm{d} J 14.3, \mathrm{H}-5), 2.77(1 \mathrm{H}, \mathrm{d} J 5.2, \mathrm{H}-$ 17), 2.48 (1H, dd $J 15.4,6.2, \mathrm{H}-22), 2.20$ (1H, obs. d $J 15.8, \mathrm{H}-2), 2.15$ (1H, dd $J$ 15.4, 4.2, H-22), 2.10 (1H, obs. d $J$ 15.8, H-2), 1.92 (1H, d J 11.1, H-16), 1.82 (1H, ddd $J$ 11.1, 5.2, 4.7, H-16), 1.77 (3H, s 18-Me), 1.51 (3H, s, 30-Me), 0.88 (9H, s, TBS), 0.12 (3H, s, TBS), -0.01 (3H, s, TBS); $\delta_{\mathrm{C}}\left(150 \mathrm{MHz}, \mathrm{CDCl}_{3}\right) 209.1$ (s), 173.8 (s), 171.1 (s), 140.7 (s), 138.3 (s), 137.7 (s), 137.0 (s), $128.5(2 \times \mathrm{d}), 128.3(2 \times \mathrm{d})$, 127.8 (d), 127.7 ( $2 \times$ d), 127.3 (d), 126.9 (2× d), 108.9 (d), 106.0 (s), 104.3 (d), 87.1 (s), $81.4(\mathrm{~d}), 75.6(\mathrm{~d}), 72.8(\mathrm{~d}), 72.5(\mathrm{t}), 68.7$ (d), $68.5(\mathrm{~d}), 65.7$ (t), 64.8 (t), 58.0 (d), 56.4 (q), 53.7 (s), 53.2 (q), 53.1 (s), 52.8 (d), 52.1 (q), 47.8 (s), 39.1 (t), 38.4 (d), 38.3 $(\mathrm{t}), 35.5(\mathrm{t}), 25.8(3 \times \mathrm{q}), 20.4(\mathrm{q}), 18.0(\mathrm{~s}), 17.4(\mathrm{q}),-5.1(\mathrm{q}),-5.3(\mathrm{q})$; HRMS 927.3931[(M+Na) ${ }^{+} \mathrm{C}_{49} \mathrm{H}_{64} \mathrm{O}_{15} \mathrm{SiNa}$ requires 927.3963] $\Delta=3.5 \mathrm{ppm}$. 


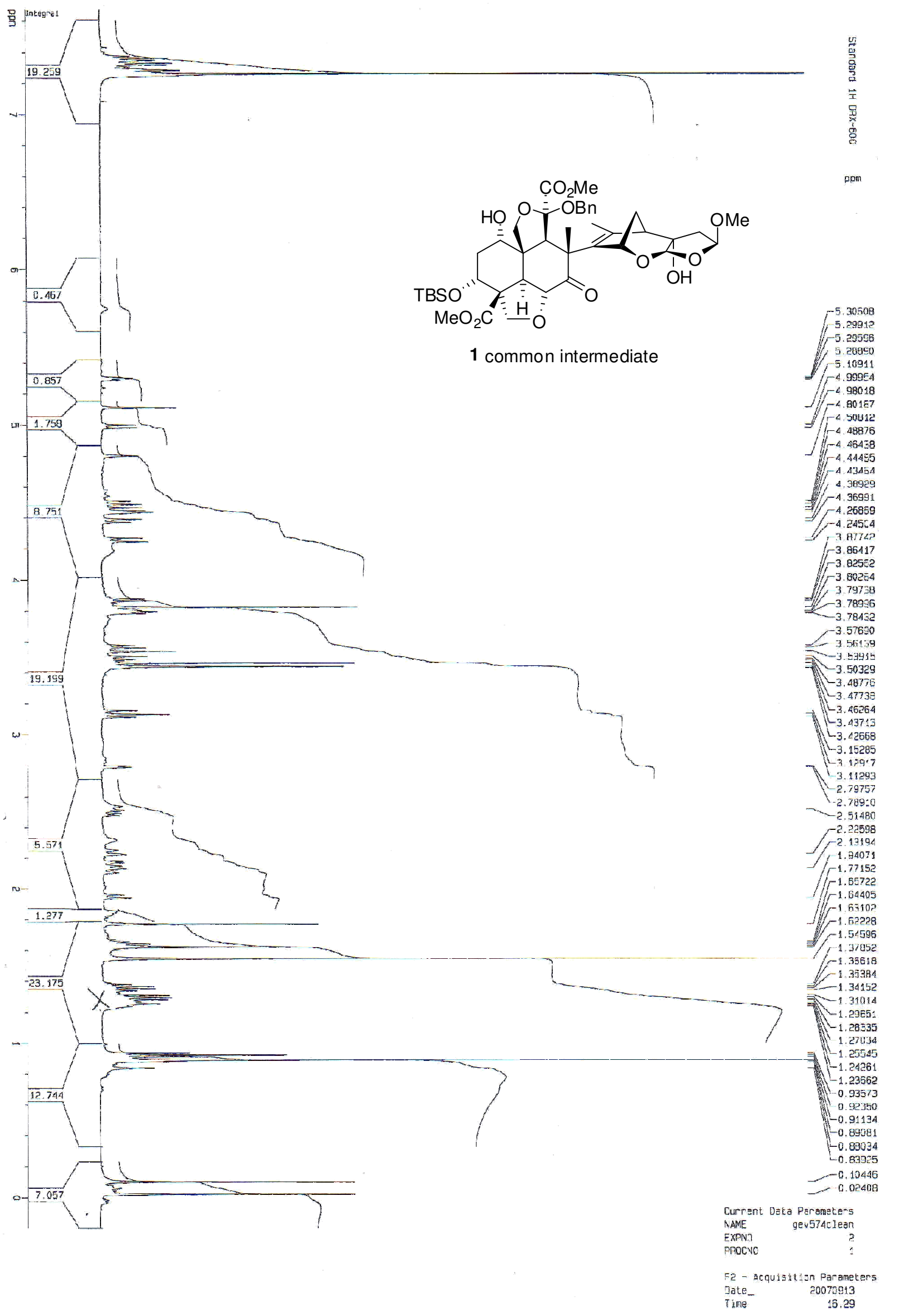




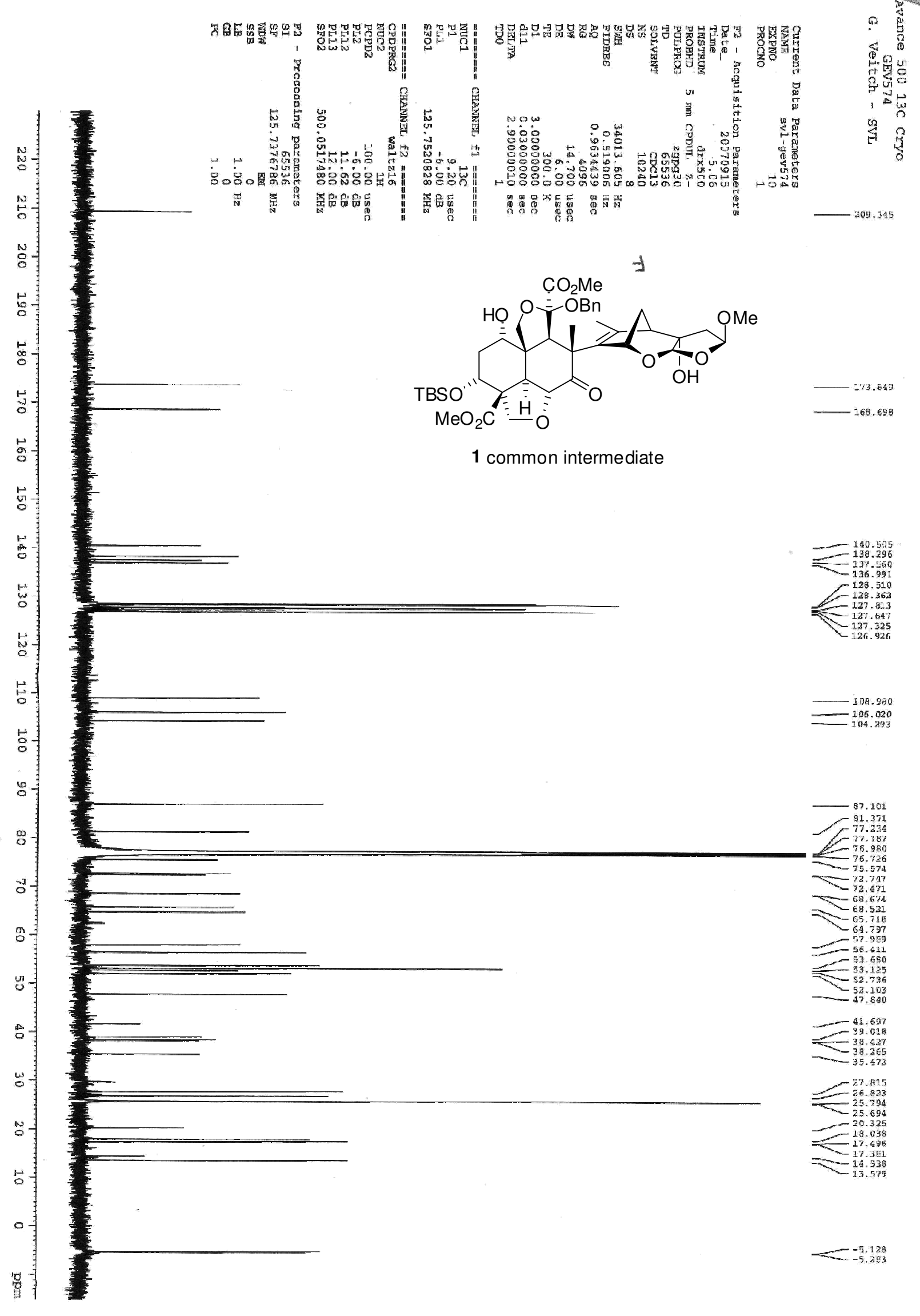




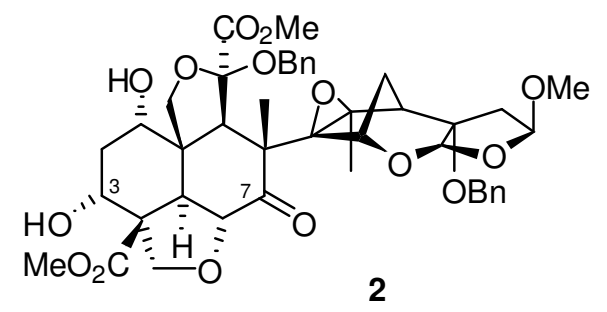

To a stirred solution of alkene $1(11 \mathrm{mg}, 0.012 \mathrm{mmol})$ in methanol (1 mL) was added magnesium monoperoxyphthalate heptahydrate (119 mg, $0.24 \mathrm{mmol}), \mathrm{NaHCO}_{3}$ (20 mg, $0.24 \mathrm{mmol}$ ), and 5-tert-butyl-4-hydroxy-2-methyl phenylsulfide ( $86 \mathrm{mg}, 0.24$ mmol). The resulting mixture was then heated in a sealed tube for 10 days at $110{ }^{\circ} \mathrm{C}$. The reaction was partitioned between ethyl acetate $(5 \mathrm{~mL})$ and sat. aq. sodium thiosulfate solution $(5 \mathrm{~mL})$. The organic layer was separated and further washed $(3 \times 10 \%$ aq. $\mathrm{NaOH}$ soln. $)$ then dried $\left(\mathrm{MgSO}_{4}\right)$ and concentrated in vacuo. The crude product was purified by flash column chromatography (80\% ethyl acetate/hexane) to afford $2(7.1 \mathrm{mg}, 81 \%)$ as a colourless oil. $[\alpha]_{\mathrm{D}}=+11.8\left(c 0.001, \mathrm{CHCl}_{3}\right) ; v_{\max } / \mathrm{cm}^{-1}$ 3456, 2953, 1753, 1725, 1440, 1286, 1092; $\delta_{\mathrm{H}}\left(600 \mathrm{MHz}, \mathrm{CDCl}_{3}\right)$ 7.36-7.23 $(10 \mathrm{H}$, Ph), 5.50 (1H, s, H-21), 5.23 (1H, dd J 6.4, 2.6, H-23), 5.08 (1H, br s, H-15), 4.99 (1H, d $J$ 12.0, H-19), 4.51 (1H, d $J$ 12.6, CHHPh), 4.49-4.47 (2H, H-19 + H-1), 4.43 (1H, d $J$ 12.5, CHHPh), 4.21 (1H, d $J 14.0, \mathrm{H}-6), 4.04$ (1H, d $J 8.2, \mathrm{H}-28), 3.95$ (2H, $\mathrm{H}-28+\mathrm{H}-3), 3.88$ (1H, d $J$ 14.4, H-5), 3.86-3.83 (5H, $\left.\mathrm{CO}_{2} \mathrm{Me}, \mathrm{H}-9+\mathrm{H}-17\right), 3.76$ $\left(1 \mathrm{H}, \mathrm{d} J\right.$ 9.6, CHHPh), $3.54(1 \mathrm{H}, \mathrm{d} J 9.6, \mathrm{CH} H \mathrm{Ph}), 3.47\left(3 \mathrm{H}, \mathrm{s}, \mathrm{CO}_{2} \mathrm{Me}\right), 3.39$ (3H, s, OMe), 2.78 (1H, d J 6.3, OH), 2.64 (1H, d J 5.8, OH), 2.49 (1H, dd $J 15.1,2.6, \mathrm{H}-22)$, 2.24 (2H, m, H-2), 2.12 (1H, dd $J$ 15.1, 6.4, H-22), 1.82 (3H, s, 18-Me), 1.64 (1H, d $J$ 12.6, H-16), 1.57 (3H, s, 30-Me), $1.52(1 \mathrm{H}, \mathrm{m}, \mathrm{H}-16)$; $\delta_{\mathrm{C}}\left(150 \mathrm{MHz}, \mathrm{CDCl}_{3}\right) 206.2$ (s), $174.2(\mathrm{~s}), 169.0$ (s), 138.1 (s), 136.9 (s), $128.5(2 \times \mathrm{d}), 128.4(2 \times \mathrm{d}), 127.8$ (d), $127.7(2 \times \mathrm{d}), 127.4$ (d), $126.9(2 \times \mathrm{d}), 107.7$ (d), 105.8 (s), 103.9 (d), 86.9 (s), 78.0 (d), $75.3(\mathrm{~d}), 73.6(\mathrm{~d}), 72.9(\mathrm{t}), 69.3(\mathrm{t}), 68.3(\mathrm{~s}), 67.4(\mathrm{~d}), 67.0(\mathrm{~s}), 65.9(\mathrm{t}), 64.4(\mathrm{t})$, 55.5 (q), 53.5 (s), 53.0 (q), 52.7 (s), 51.9 (q), 48.7 (s), 46.9 (d), 41.3 (t), 37.7 (d), 35.6 (d), 35.5 (t), 25.1 (t), 20.0 (q), 16.5 (q); HRMS 829.3040 [(M+Na) ${ }^{+} \mathrm{C}_{43} \mathrm{H}_{50} \mathrm{O}_{15} \mathrm{Na}$ requires 829.3047$] \Delta=0.9 \mathrm{ppm}$. 


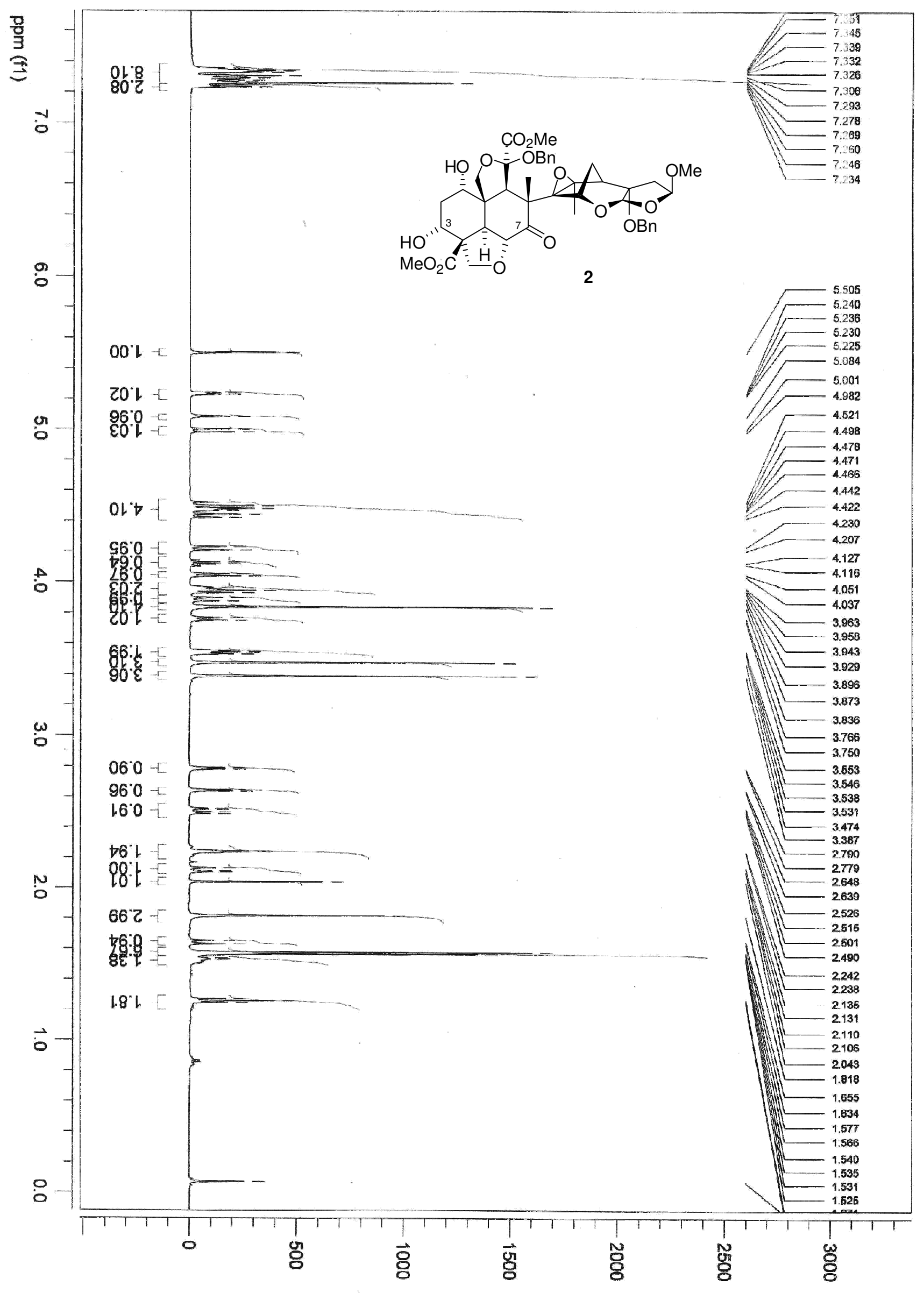




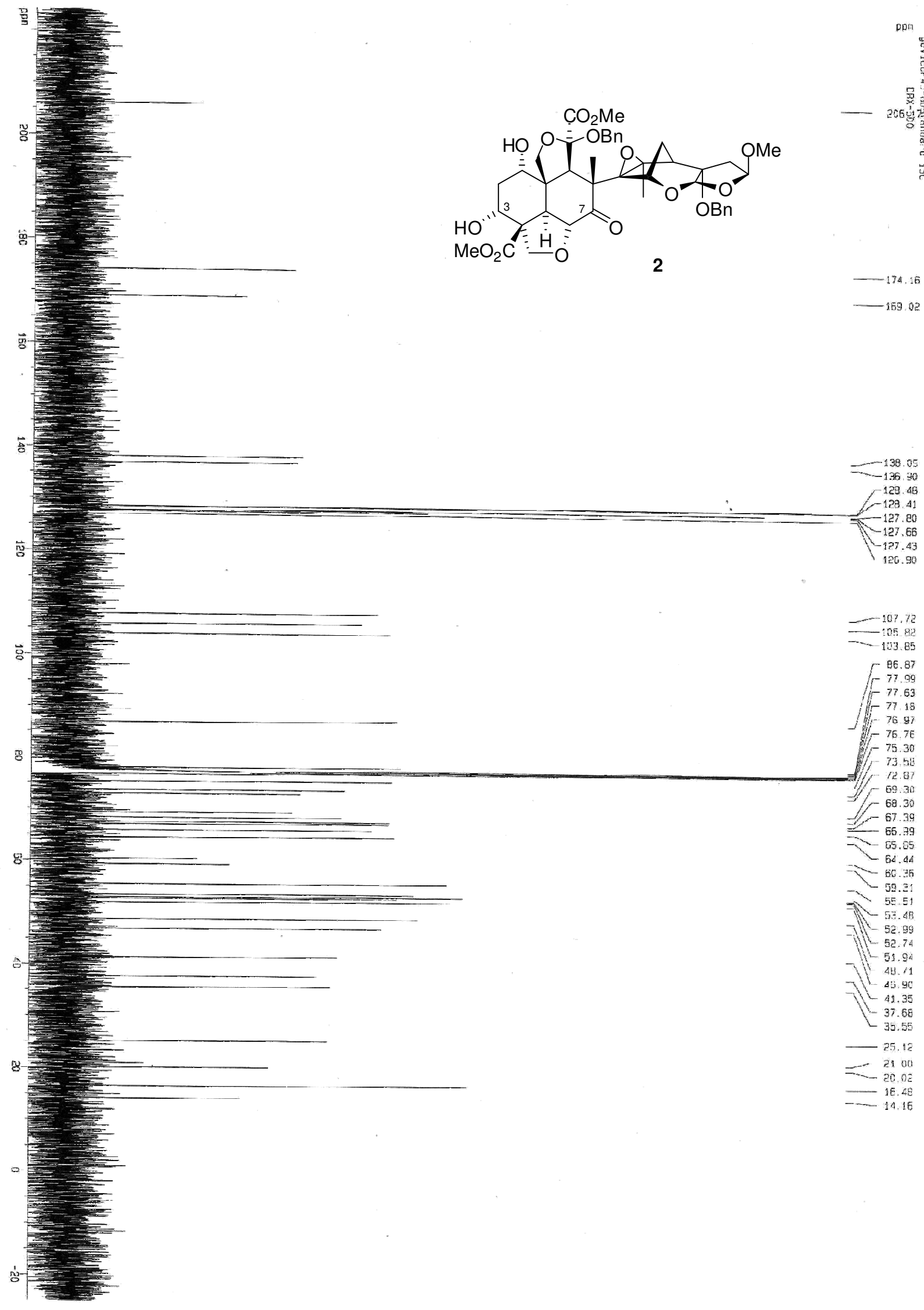


Vepaol 3

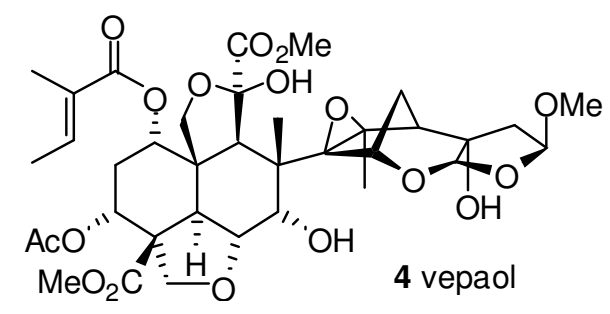

$v_{\max } / \mathrm{cm}^{-1} 3442,2921,1735,1437,1376,1266 ; \delta_{\mathrm{H}}\left(400 \mathrm{MHz}, \mathrm{CDCl}_{3}\right) 6.86(1 \mathrm{H}, \mathrm{q} J$ 7.1, H-3'), 5.49 (1H, obs. t J 3.0, H-3), 5.48 (1H, s, H-21), 5.17 (1H, dd J 6.3, 5.2, H23), $5.03(1 \mathrm{H}, \mathrm{s}, \mathrm{OH}), 4.73(1 \mathrm{H}$, obs. t J 2.7, H-1), $4.67(2 \mathrm{H}, \mathrm{H}-7+\mathrm{H}-15), 4.58(1 \mathrm{H}$, dd $J 12.5,2.7, \mathrm{H}-6), 4.15$ (1H, d $J 9.7, \mathrm{H}-19), 4.06$ (1H, d $J 9.0, \mathrm{H}-28), 3.78$ (3H, s, $\left.\mathrm{CO}_{2} \mathrm{Me}\right), 3.73(1 \mathrm{H}, \mathrm{d} J 9.0, \mathrm{H}-19), 3.68\left(3 \mathrm{H}, \mathrm{s}, \mathrm{CO}_{2} \mathrm{Me}\right), 3.61(1 \mathrm{H}, \mathrm{d} J 9.8, \mathrm{H}-28)$, 3.42 (3H, s, OMe), 3.29 (1H, s, H-9), 3.24 (1H, d J 12.5, H-5), 2.81 (1H, s, OH), 2.55 (1H, s, OH), 2.47 (1H, d J 5.3, H-17), 2.38 (1H, dd J 14.7, 6.4, H-22), 2.32 (1H, obs. dt $J$ 17.0, 2.4, H-2), 2.24-2.18 (2H, H-2 + H-22), 2.00 (3H, s, 18-Me), 1.94 (3H, s, 3OAc), 1.91 (1H, d J 8.2, H-16), 1.84 (3H, s, 5'-Me), 1.77 (3H, d J 7.1, 4'-Me), 1.74 (3H, s, 30-Me) $1.63(1 \mathrm{H}, \mathrm{m}, \mathrm{H}-16)$; $\delta_{\mathrm{C}}\left(150 \mathrm{MHz}, \mathrm{CDCl}_{3}\right) 173.3$ (s), 171.7 (s), 169.6 (s), 166.2 (s), 137.6 (d), 128.6 (s), 106.7 (d), 105.9 (d), 104.2 (s), 81.0 (s), 77.1 (d), 74.0 (d), 73.9 (d), 72.9 (t), 70.5 (d), $69.4(\mathrm{~s}), 69.0$ (t), 68.5 (s), 66.9 (d), 55.7 (q), 53.1 (q), $52.7(\mathrm{q}), 52.4(\mathrm{~s}), 50.0(\mathrm{~s}), 49.9(\mathrm{~d}), 48.0(\mathrm{t}), 45.3(\mathrm{~s}), 44.7(\mathrm{~d}), 37.1(\mathrm{~d}), 29.7(\mathrm{t})$, 24.7 (t), 21.3 (q), 20.8 (q), 18.4 (q), 14.3 (q), 11.9 (q); HRMS 753.2979 [(M+H) ${ }^{+}$ $\mathrm{C}_{36} \mathrm{H}_{49} \mathrm{O}_{17}$ requires 753.2970$] \Delta=1.2 \mathrm{ppm}$. 


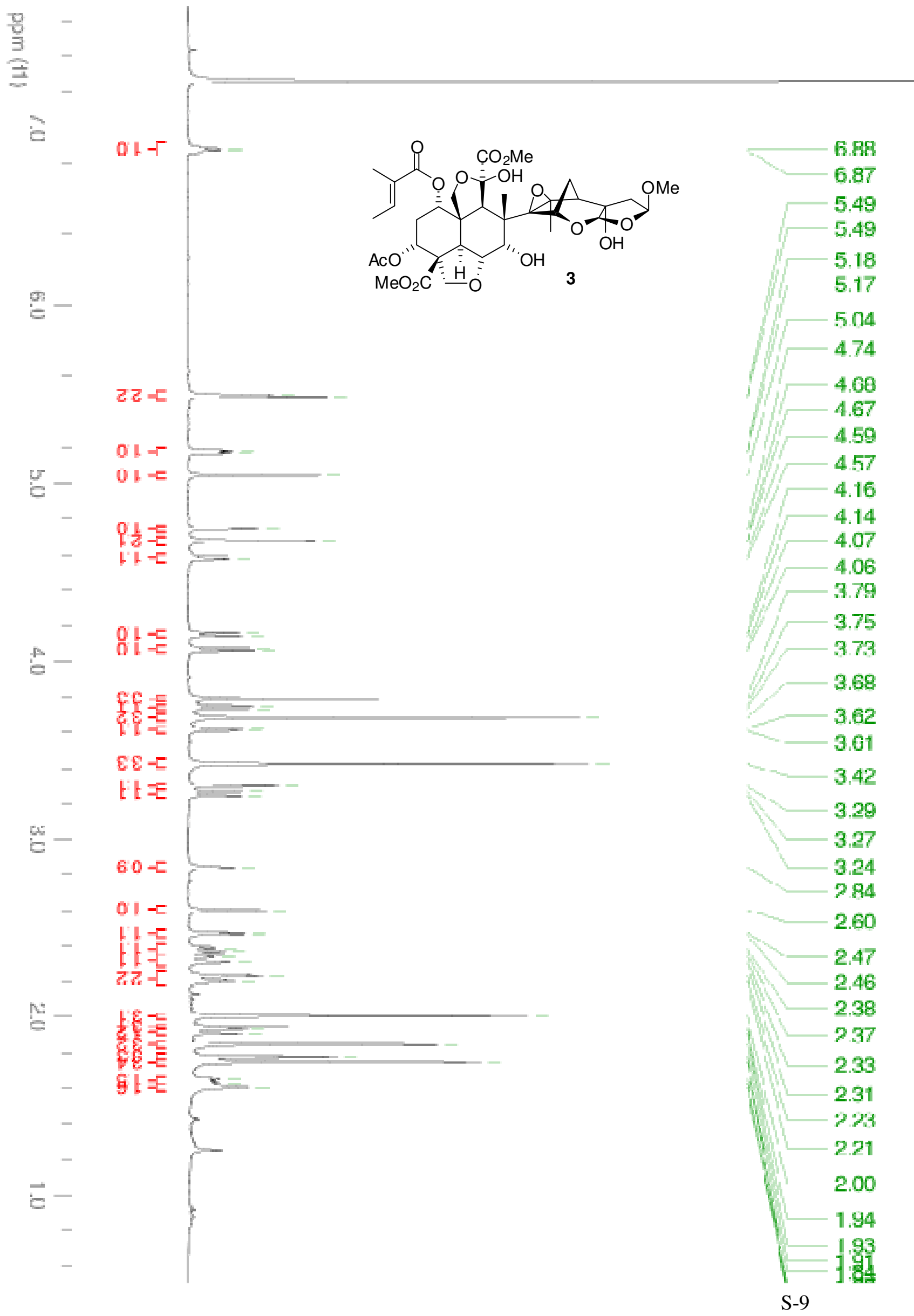




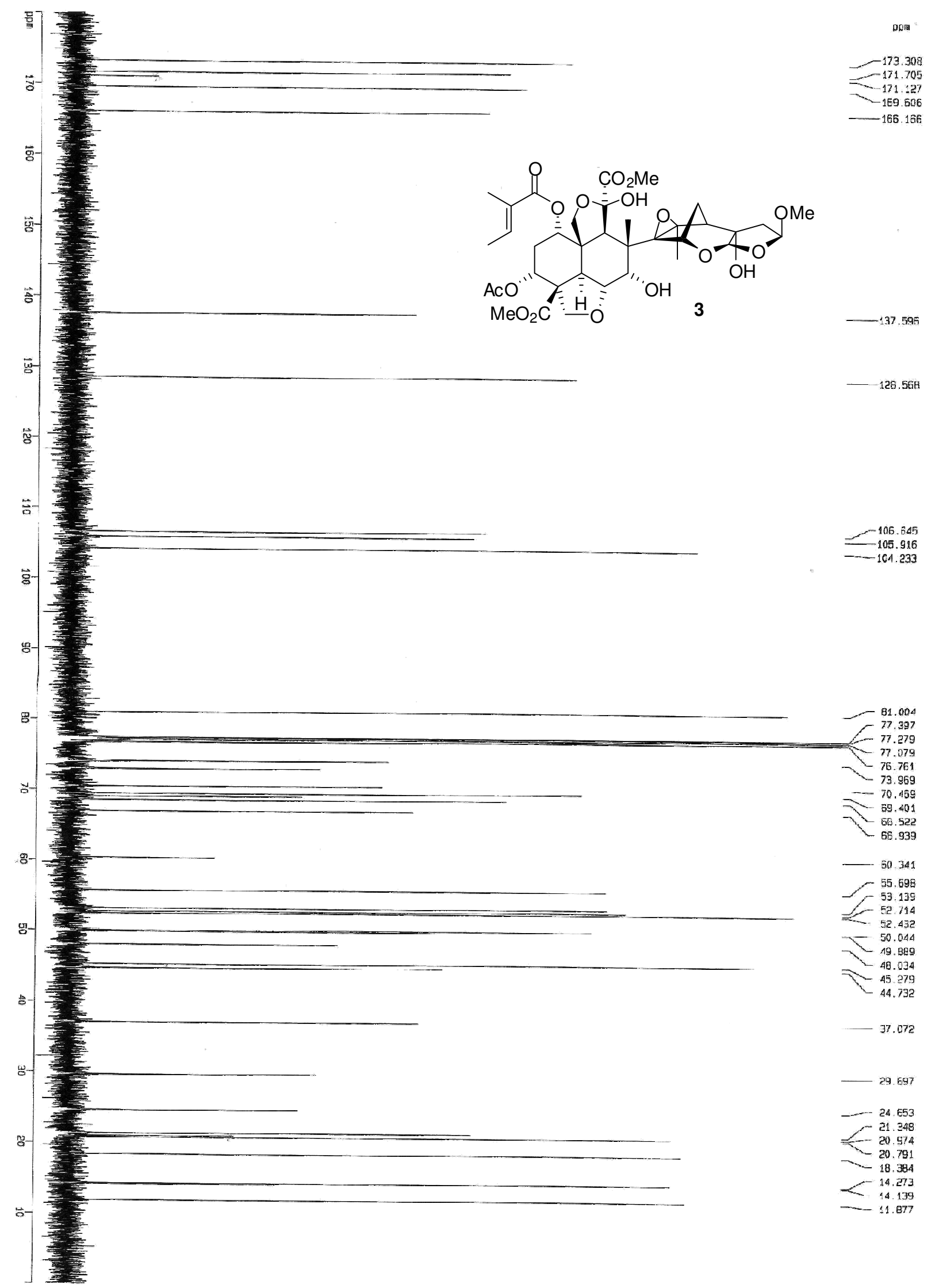

S-10 


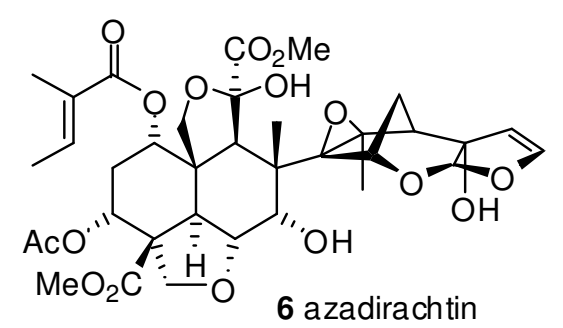

To a stirred solution of vepaol $3(50 \mathrm{mg}, 0.066 \mathrm{mmol})$ in 1,2 dichloroethane $(2.5 \mathrm{~mL})$ was added benzeneselenol (150 $\mu \mathrm{L}, 1.33 \mathrm{mmol})$ followed by PPTS (cat., 5 crystals). The resulting mixture was heated at reflux for $4 \mathrm{~h}$, then loaded directly onto silica and chromatographed with ethyl acetate/hexane (20-50\%) to afford 5 (59 mg, quant.) as a highly unstable yellow oil.

Selenide 5 (59 mg, $0.066 \mathrm{mmol})$ was dissolved in dichloromethane $(2 \mathrm{~mL})$ and cooled to $0{ }^{\circ} \mathrm{C}$. Pyridine $(27 \mu \mathrm{L}, 0.33 \mathrm{mmol})$ followed by hydrogen peroxide $(30 \%$ aq. soln., $37 \mu \mathrm{L}, 0.33 \mathrm{mmol}$ ) was then added and the resulting mixture stirred for $10 \mathrm{~min}$. The reaction was then quenched via addition of sat. aq. sodium thiosulfate soln. $(1 \mathrm{~mL})$ and the organic layer separated, dried $\left(\mathrm{MgSO}_{4}\right)$ and concentrated in vacuo. Column chromatography (80\% ethyl acetate/hexane) afforded azadirachtin 6 (41 mg, 85\%) as a white amorphous solid (41 mg, 85\%). $v_{\max } / \mathrm{cm}^{-1} 3441,2923,1741,1438,1376$, 1265; $\delta_{\mathrm{H}}\left(400 \mathrm{MHz}, \mathrm{CDCl}_{3}\right) 6.92(1 \mathrm{H}, \mathrm{q} J$ 7.1, H-3'), 6.46 (1H, d J 2.9, H-23), 5.64 (1H, s, H-21), $5.50(1 \mathrm{H}$, obs. t J 2.8, H-3), 5.05 (1H, d J 2.8, H-22), $5.02(1 \mathrm{H}, \mathrm{s}, \mathrm{OH})$, $4.76(1 \mathrm{H}$, obs. t $J$ 2.8, H-1), $4.74(1 \mathrm{H}$, br s, H-7), $4.67(1 \mathrm{H}, \mathrm{d} J 3.5, \mathrm{H}-15), 4.61(1 \mathrm{H}$, dd $J$ 12.5, 2.7, H-6), 4.15 (1H, d $J 9.7, \mathrm{H}-19), 4.07$ (1H, d $J 9.0, \mathrm{H}-28), 3.79$ (3H, s, $\left.\mathrm{CO}_{2} \mathrm{Me}\right), 3.77\left(1 \mathrm{H}, \mathrm{d} J\right.$ 9.0, H-28), $3.69\left(3 \mathrm{H}, \mathrm{s}, \mathrm{CO}_{2} \mathrm{Me}\right), 3.63(1 \mathrm{H}, \mathrm{d} J 9.7, \mathrm{H}-19)$, 3.35 (1H, d J 12.4, H-5), 3.34 (1H, s, H-9), 2.84 (1H, br s, OH), $2.79(1 \mathrm{H}, \mathrm{br} \mathrm{s}, \mathrm{OH})$, 2.38 (1H, d $J$ 5.3, H-17), 2.34 (1H, obs. dt $J$ 16.9, t not resolved, H-2), 2.23 (1H, obs. dt $J$ 16.9, 3.3, H-2), 2.00 (3H, s, 18-Me), 1.95 (3H, s, 3-OAc), 1.85 (3H, s, 5'-Me), 1.78 (3H, d $J$ 7.1, 4'-Me), 1.75 (3H, s, 30-Me), 1.76 (1H, ddd $J 13.2,5.3,3.5, \mathrm{H}-16)$, 1.31 (1H, ddd $J$ 13.2, 0.4, 0.3, H-16); $\delta_{\mathrm{C}}\left(100 \mathrm{MHz}, \mathrm{CDCl}_{3}\right) 173.4$ (s), 171.8 (s), 169.7 (s), 166.2 (s), 146.9 (d), 137.8 (d), 128.5 (s), 108.6 (d), 107.5 (d), 104.2 (s), 83.5 (s), 76.4 (d), 74.3 (d), 73.9 (d), 73.1 (t), 70.5 (d), 70.0 (s), 68.0 (t), 67.5 (s), 66.9 (d), 53.2 (q), 52.7 (q), 52.5 (s), 50.2 (s), 48.6 (d), 45.5 (s), 44.7 (d), 36.9 (d), 29.7 (t), 25.0 (t), $21.3(\mathrm{q}), 20.8(\mathrm{q}), 18.4(\mathrm{q}), 14.3(\mathrm{q}), 11.9$ (q). 


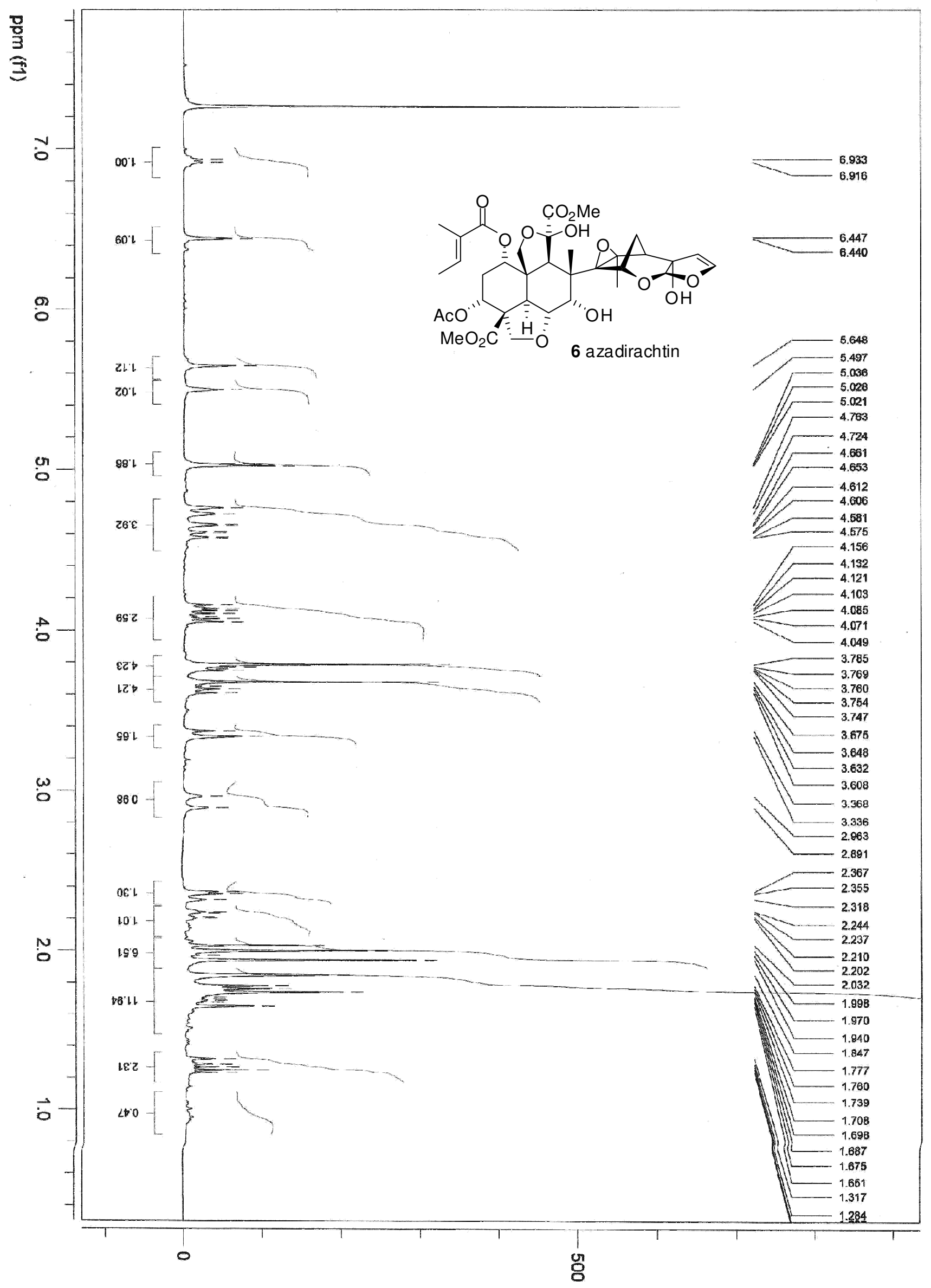




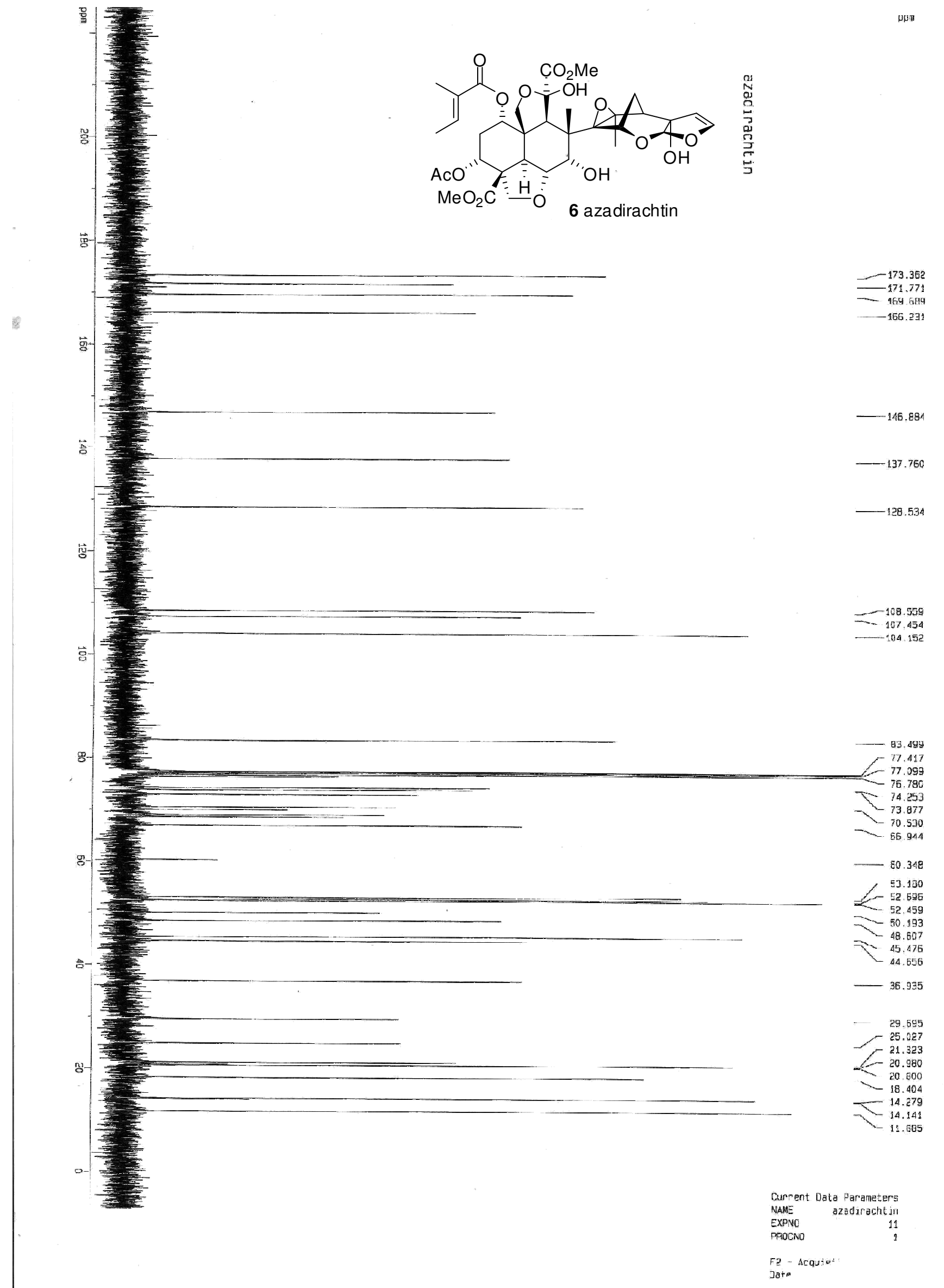

